\title{
A REVIEW OF CERVICAL SPINE INJURIES WITH NEUROLOGICAL DYSFUNCTION
}

\author{
By G. M. Bedbrook, K.B., O.B.E., O.StJ., Hon. M.D. (W.A.), M.S. \\ (Melb.), D.P.R.M., F.R.C.S., F.R.A.C.S., F.R.C.S. (Edin.) Hon. ${ }^{1}$ and \\ T. SAKAE, M.D. ${ }^{2}$ \\ 1 Senior Surgeon to Spinal Unit, Royal Perth (Rehabilitation) Hospital. Orthopaedic \\ Surgeon, Royal Perth and Royal Perth (Rehabilitation) Hospitals. Formally Director \\ of Spinal Unit and Orthopaedic Unit, Royal Perth and Royal Perth (Rehabilitation) \\ Hospital. ${ }^{2}$ Research Fellow to Royal Perth (Rehabilitation) Hospital, Spinal Unit, \\ Orthopaedic Surgeon. National Kumamoto Hospital, fapan.
}

\begin{abstract}
The authors have studied 193 cases of cervical injury with tetraparesis or paralysis in a similar way to a previous study completed in 1973 (Bedbrook 1973). Comparison with three other centres, Stoke Mandeville, Tokushima and South West Region has shown similarities and regional dissimilarities-for example in aetiology.

Only in cases with bilateral facet dislocation was reduction considered to have any effect on neurological recovery in all four geographical areas. The observations recorded strengthen the view that the result of the neurological injury is largely determined at the time of the accident.
\end{abstract}

Key words: Cervical spine injury; Neurological dysfunction; Biomechanics; Reduction; Outcome.

\section{Introduction}

IN 1973 at the I9th Veterans Administration Spinal Cord Injury Conference in Phoenix, Arizona, the senior author of this study presented a review of 236 cases manifesting cervical injury with neurological dysfunction treated in Perth between the years I95 I to I973 (Bedbrook I973).

The present study extends that review and makes observations on neurological changes in a similar way to Frankel and his colleagues at that conference, who also reported on such work (Frankel et al., I969, 1977).

As in the previous review, all patients with tetraparesis and tetraplegia from cervical injury were basically treated by postural reduction. This is an old but well tried proven method. Once diagnosis has been made, even before the finalisation of detail, these methods should be immediately undertaken in any hospital. They do not call for expensive equipment. Change of postural position every two hours is essential. In those injuries where such maintenance was difficult, skeletal traction was then used before other methods. The 'Egerton-Stoke Mandeville' bed has introduced greater ease in this method and is in the opinion of the present authors a most satisfactory orthosis. Detailed discussion of postural care between physician and nursing staff is mandatory. Such a method is of greater importance than any technical surgical procedure. With correct posture, many fractures will reduce and be stable, provided maintenance is then good for 6 to 8 weeks. Those who need further reduction can be treated by other appropriate non-operative methods including skeletal 
traction with manipulation and restitution with or without general anaesthesia.

\section{Material}

In the period July I973 to December I980, a total of I93 cases with cervical spinal injuries were admitted to the Spinal Unit at the Royal Perth (Rehabilitation) Hospital.

All patients with upper cervical injuries $\left(\mathrm{C}_{1}, \mathrm{C}_{2}\right)$ and patients who died in the first three months or who were not admitted within I 4 days were excluded from this series to make two series comparable and because at two weeks, early healing can already be occurring. This left a total of I44 patients where results could be analysed in depth.

Of these, according to the previous classification:

(a) 48 cases $(33 \%)$ sustained compression injuries

(b) 72 cases $(50 \%$ ) flexion rotation injuries

(c) 24 cases $(17 \%$ ) extension injuries

(see Table I)

Data was collected from a number of sources; patient records, X-ray records, patient interviews allowing further clinical and radiological investigation as required.

\section{TABLE I}

Review of Cervical Fractures and Fracture Dislocations Perth, Western Australia

\begin{tabular}{|c|c|c|c|}
\hline & I95I-73 & I973-80 & I 95 I-80 \\
\hline Compression & $6326 \%$ & $4833 \%$ & I I I $29 \%$ \\
\hline Flexion Rotation & I 2 I $48 \%$ & $7250 \%$ & $19349 \%$ \\
\hline Extension & $6326 \%$ & $2417 \%$ & $8722 \%$ \\
\hline Total & $247100 \%$ & $144100 \%$ & $391100 \%$ \\
\hline
\end{tabular}

\section{Age}

Almost all the patients were aged between twenty and thirty years (see Table II). Half of the patients over fifty years of age, manifested an extension injury. Burke (I97I) reported that 29 out of his 67 extension injuries were below the age of 45 years and 38 were above the age of 45 years. This is thus not exclusively a domestic accident of elderly people. In our series, ten out of 24 patients with extension injuries were below the age of 45 years and 14 above the age of 45 years.

\section{Cause}

The commonest cause of injury was car accidents, driver and passenger inclusive $(56 \%)$. Sports accidents, mainly diving and surfing, accounted for $24 \%$. It is clear that the cause of accidents varies greatly from one area to another. 


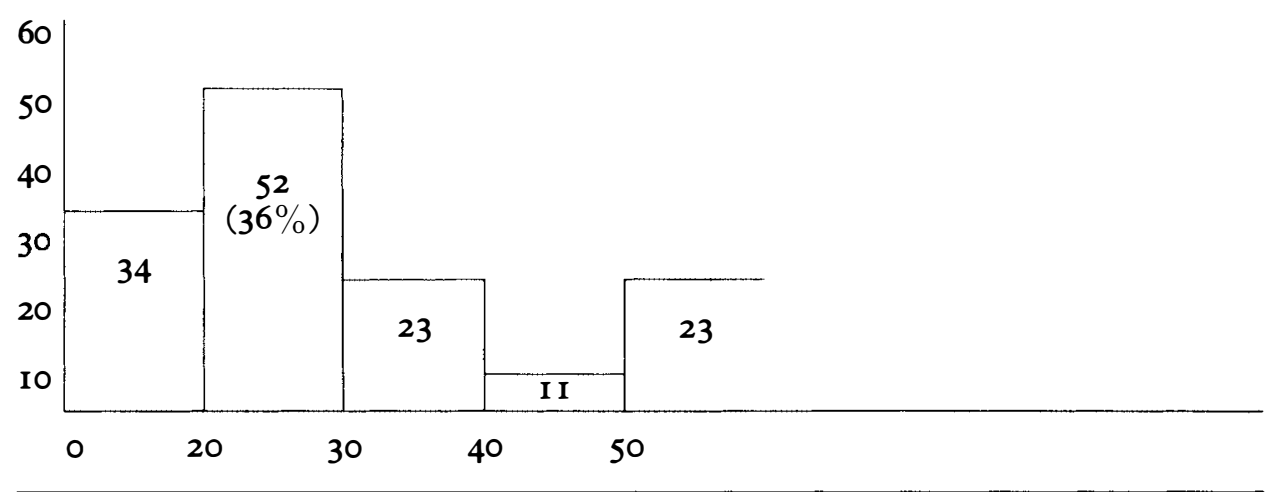

Period of Time Before Admission to Hospital

$84 \%$ of the patients were transferred to hospital within 24 hours whilst $30 \%$ were transferred within 3 hours (see Table III).

\section{Neurological Change}

The present authors used Frankel's grid method to classify the neurological status.

(A) Complete: This indicates that the lesion was found to be complete TABLE III

Period of Time Before Admission

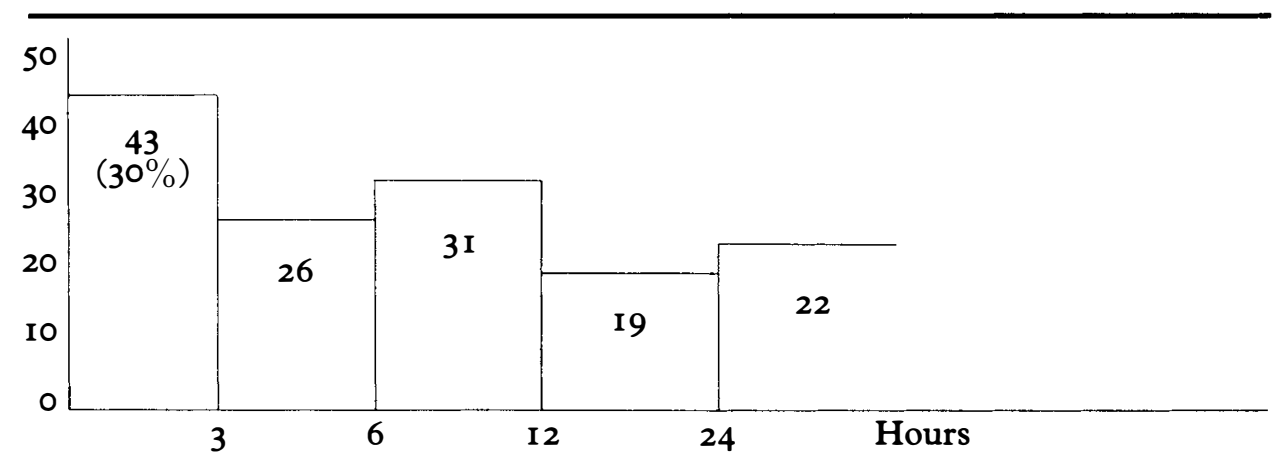

Period of Time Spent in Bed and Hospital

Compression

Flexion Rotation I

II
Period in Bed

33 days

37 days

4 I days

22 days
Period in Hospital

I 52 days

88 days

I 49 days

87 days 
both motor and sensory below the level of the lesion. If there was an alteration of level but the lesion remained 'complete' below the new level, the patient was considered to have remained 'complete'.

(B) Sensory Sparing Only: This implies that there was some sensation present below the level of the lesion but that the motor paralysis was complete below that level.

(C) Motor Useless: This implies that there was some motor power below the level of the lesion but it was of no practical use to the patient.

(D) Motor Useful: This implies that there was useful motor power below the level of the lesion. Patients in this group could move the lower limbs and many could eventually walk, with or without aids.

(E) Recovery: This implies that the patient was free of neurological symptoms.

In each table the 'Frankel Grade' is documented at the time of admission and recorded in the appropriate row labelled $\mathrm{A}$ through $\mathrm{E}$ at the left of the grid. The grade at discharge is recorded in the appropriate column labelled $\mathrm{A}$ through $\mathrm{E}$ above and to the right of the grid. The numbers of cases whose neurological status remained unchanged are represented with diagonal cells outlined in heavy lines. Cells above and to the right of the heavy line contain cases which improved. Those cases below and to the left of the heavy line are cases that deteriorated. Data on neurological status on admission and discharge for all cases in this study, along with comparable data reported by Stoke Mandeville, South West Region and tokushima University (Japan) are repesented in Tables IV and V. Percentage of improvement in each group is shown on the left and right sides of the tables.

The improvement of each group at the Royal Perth (Rehabilitation) Hospital were (A 36\%, B 61\%, C $87 \%$ and D $86 \%$ ) almost similar to the Stoke Mandeville results. Apart from group A, the South West Region gained almost the same result, (see Table IV). The Tokushima results varied a little probably due to causal facts, see later.

Operations, including anterior and posterior surgery for reduction, and/ or fusions were undertaken in;

(a) $10 \%$ at the Royal Perth (Rehabilitation) Hospital (anterior fusion six cases, open reduction eight cases, laminectomy one case).

(b) $0 \%$ in the Stoke Mandeville Hospital.

(c) $39 \%$ in the South West Region (anterior fusion 26 cases, laminectomy nine cases).

(d) $51 \%$ in Tokushima (Japan) (anterior fusion 52 cases, laminectomy one case, anterior fusion and laminectomy three cases).

The authors extended the study to include observations on useful recovery (see Tables VII, IXa and IXb).

Within the useful recovery of the group only $2.6 \%$ (one case) of the Royal Perth (Rehabilitation) Hospital patients showed return of motor function (see Table V). Only 9\% (I I cases) from the Stoke Mandeville Hospital, $2 \%$ (one case) from the South West Region showed this degree of 
TABLE IV

Comparison of Neurological Improvement (Cervical Injuries)

\begin{tabular}{|c|c|c|c|c|c|}
\hline \multirow[b]{2}{*}{$36 \%$} & \multicolumn{5}{|c|}{ R.P.(R.)H. $\mathrm{n}=\mathrm{I} 44$} \\
\hline & AA & $\begin{array}{c}\mathrm{AB} \\
\text { I0 }\end{array}$ & $\mathrm{AC}$ & $\underset{\mathbf{I}}{\mathrm{AD}}$ & $\mathrm{AE}$ \\
\hline $6 \mathrm{r} \%$ & $\mathrm{BA}$ & $\overline{\mathrm{BB}}$ & $\begin{array}{c}\mathrm{BC} \\
4\end{array}$ & $\begin{array}{c}\mathrm{BD} \\
7\end{array}$ & $\mathrm{BE}$ \\
\hline $87 \%$ & CA & $\begin{array}{c}\text { CB } \\
\text { I }\end{array}$ & $\begin{array}{c}\mathrm{CC} \\
2\end{array}$ & $\begin{array}{c}\mathrm{CD} \\
\mathrm{I} 5 \\
\end{array}$ & $\begin{array}{c}C E \\
5\end{array}$ \\
\hline $86 \%$ & $\mathrm{DA}$ & $\begin{array}{c}\text { DB } \\
\text { I }\end{array}$ & $\overline{\mathrm{DC}}$ & $\begin{array}{c}\mathrm{DD} \\
7\end{array}$ & $\begin{array}{c}\text { DE } \\
48 \\
\end{array}$ \\
\hline & EA & EB & $\mathrm{EC}$ & ED & $\begin{array}{c}\mathrm{EE} \\
8\end{array}$ \\
\hline
\end{tabular}

South West Region $\mathrm{n}=95$

\begin{tabular}{|c|c|c|c|c|c|}
\hline $16.3 \%$ & $\begin{array}{l}\text { AA } \\
4 I\end{array}$ & $\begin{array}{c}\mathrm{AB} \\
6 \\
\end{array}$ & $\begin{array}{c}\text { AC } \\
\text { I }\end{array}$ & $\begin{array}{c}\mathrm{AD} \\
\mathrm{I}\end{array}$ & $\mathrm{AE}$ \\
\hline $75 \%$ & $\overline{\mathrm{BA}}$ & $\begin{array}{c}\mathrm{BB} \\
4\end{array}$ & $\begin{array}{c}\mathrm{BC} \\
2\end{array}$ & $\begin{array}{c}\mathrm{BD} \\
\text { I0 }\end{array}$ & $\mathrm{BE}$ \\
\hline $60 \%$ & $\mathrm{CA}$ & $\overline{\mathrm{CB}}$ & $\begin{array}{c}\mathrm{CC} \\
2\end{array}$ & $\begin{array}{c}C D \\
3\end{array}$ & $\mathrm{CE}$ \\
\hline \multirow[t]{2}{*}{$30 \%$} & DA & DB & $\overline{\mathrm{DC}}$ & $\begin{array}{c}\mathrm{DD} \\
\mathrm{I} 4\end{array}$ & $\begin{array}{c}\mathrm{DE} \\
6\end{array}$ \\
\hline & EA & EB & $\mathrm{EC}$ & $\begin{array}{c}\text { ED } \\
3\end{array}$ & $\overline{\mathrm{EE}}$ \\
\hline
\end{tabular}

Stoke Mandeville $\mathrm{n}=2 \mathrm{I} 8$

\begin{tabular}{|c|c|c|c|c|}
\hline $\begin{array}{c}\text { AA } \\
8 \mathrm{I}\end{array}$ & $\begin{array}{c}\mathrm{AB} \\
2 \mathrm{I}\end{array}$ & $\begin{array}{c}\text { AC } \\
\text { IO }\end{array}$ & $\begin{array}{c}\text { AD } \\
\text { I I }\end{array}$ & $\mathrm{AE}$ \\
\hline $\mathrm{BA}$ & $\mathrm{BB}$ & BC & $\mathrm{BD}$ & $\mathrm{BE}$ \\
\hline 3 & 9 & 2 & 14 & 15 \\
\hline CA & $\begin{array}{c}\mathrm{CB} \\
\mathrm{I}\end{array}$ & $\begin{array}{c}\mathrm{CC} \\
4\end{array}$ & $\begin{array}{c}\text { CD } \\
\text { I I }\end{array}$ & $\begin{array}{c}\mathrm{CE} \\
5\end{array}$ \\
\hline DA & DB & $\mathrm{DC}$ & $\begin{array}{c}\mathrm{DD} \\
30\end{array}$ & $\begin{array}{c}\mathrm{DE} \\
\mathrm{I} \text { I }\end{array}$ \\
\hline EA & EB & $\mathrm{EC}$ & ED & $\mathrm{EE}$ \\
\hline
\end{tabular}

Tokushima (Japan) $\mathrm{n}=\mathrm{I} 09$

\begin{tabular}{|c|c|c|c|c|}
\hline $\begin{array}{l}\text { AA } \\
36 \\
\end{array}$ & $\begin{array}{c}\mathrm{AB} \\
\mathrm{I}\end{array}$ & $\begin{array}{c}\mathrm{AC} \\
5 \\
\end{array}$ & $\begin{array}{c}\text { AD } \\
23 \\
\end{array}$ & $\begin{array}{c}\mathrm{AE} \\
2 \\
\end{array}$ \\
\hline $\mathrm{BA}$ & $\begin{array}{c}\mathrm{BB} \\
3\end{array}$ & BC & $\begin{array}{c}\mathrm{BD} \\
4\end{array}$ & $\begin{array}{c}\mathrm{BE} \\
4\end{array}$ \\
\hline CA & $\mathrm{CB}$ & CC & $\begin{array}{c}\mathrm{CD} \\
5 \\
\end{array}$ & $\begin{array}{c}\mathrm{CE} \\
\mathbf{2}\end{array}$ \\
\hline DA & DB & $\mathrm{DC}$ & $\begin{array}{c}\text { DD } \\
8 \\
\end{array}$ & $\begin{array}{c}\mathrm{DE} \\
\mathrm{1} 6 \\
\end{array}$ \\
\hline EA & EB & $\mathrm{EC}$ & ED & $\mathrm{EE}$ \\
\hline
\end{tabular}

recovery. On the contrary to these results, Tokushima (Japan) (in group A case) showed $37 \%$ return of motor function. For all the other comparable neurological groups, the degree of recovery of motor function observed by the three centres was almost similar but the Royal Perth (Rehabilitation) Hospital was lower at 39\%. (see Table V).

When the aetiology is compared the following was observed, Royal Perth (Rehabilitation) Hospital recorded $60.4 \%$ of all road accidents, $14.6 \%$ were caused by falls or falling objects, $24.3 \%$ because of sporting injuries, whereas in Stoke Mandeville, road accidents accounted for $49.9 \%$ of injuries, falls or falling objects $36.4 \%$ and sport $10.4 \%$. South West Region recorded the following aetiology of injuries, road accidents $63.2 \%$ falls and falling objects $20.9 \%$ and sport $12.9 \%$. Tokushima had the following results, road accidents $42.5 \%$, falls and falling objects $46.9 \%$ and sporting accidents $8 \cdot 8 \%$. 
Comparison of Neurological Improvement (Cervical Injuries) Useful Recovery

\begin{tabular}{c|c|c|c|c|c|}
\hline \multicolumn{6}{c}{ R.P.(R.)H. $\mathrm{n}=\mathrm{I} 44$} \\
\hline \multirow{3}{*}{$39 \%$} & $\mathrm{AA}$ & $\mathrm{AB}$ & $\mathrm{AC}$ & $\mathrm{AD}$ & $\mathrm{AE}$ \\
25 & $\mathrm{IO}$ & 3 & $\mathrm{I}$ & \\
\hline \multirow{3}{*}{$91 \%$} & $\mathrm{BA}$ & $\mathrm{BB}$ & $\mathrm{BC}$ & $\mathrm{BD}$ & $\mathrm{BE}$ \\
& $\mathrm{CA}$ & $\mathrm{CB}$ & $\mathrm{CC}$ & $\mathrm{CD}$ & $\mathrm{CE}$ \\
& & 2 & $\mathrm{I} 5$ & 5 \\
\cline { 2 - 7 } & $\mathrm{DA}$ & $\mathrm{DB}$ & $\mathrm{DC}$ & $\mathrm{DD}$ & $\mathrm{DE}$ \\
& & & 7 & 48 \\
\hline $\mathrm{EA}$ & $\mathrm{EB}$ & $\mathrm{EC}$ & $\mathrm{ED}$ & $\mathrm{EE}$ \\
& & & & 8 \\
\hline
\end{tabular}

South West Region $\mathrm{n}=95$

\begin{tabular}{|c|c|c|c|c|c|}
\hline $2 \%$ & $\begin{array}{c}\mathrm{AA} \\
4 \mathrm{I}\end{array}$ & $\begin{array}{c}A B \\
6 \\
\end{array}$ & $\begin{array}{c}\mathrm{AC} \\
\mathrm{I}\end{array}$ & $\begin{array}{c}\mathrm{AD} \\
\mathbf{I}\end{array}$ & $\mathrm{AE}$ \\
\hline $62.5 \%$ & $\mathrm{BA}$ & $\begin{array}{c}\text { BB } \\
4\end{array}$ & $\begin{array}{c}\mathrm{BC} \\
2\end{array}$ & $\begin{array}{c}\mathrm{BD} \\
\text { I0 }\end{array}$ & $\mathrm{BE}$ \\
\hline \multirow[t]{3}{*}{$60 \%$} & CA & $\overline{\mathrm{CB}}$ & $\begin{array}{c}\mathrm{CC} \\
2\end{array}$ & $\begin{array}{c}\mathrm{CD} \\
3\end{array}$ & $\mathrm{CE}$ \\
\hline & DA & DB & $\mathrm{DC}$ & \begin{tabular}{|} 
DD \\
I4 \\
\end{tabular} & $\begin{array}{c}\mathrm{DE} \\
6 \\
\end{array}$ \\
\hline & EA & EB & EC & $\begin{array}{c}\text { ED } \\
3\end{array}$ & $\overline{\mathrm{EE}}$ \\
\hline
\end{tabular}

Stoke Mandeville $\mathrm{n}=\mathbf{2} \mathrm{I} 8$

\begin{tabular}{|c|c|c|c|c|}
\hline $\begin{array}{c}\text { AA } \\
8 \mathrm{I} \\
\end{array}$ & $\begin{array}{l}\mathrm{AB} \\
2 \mathrm{I} \\
\end{array}$ & $\begin{array}{l}\text { AC } \\
\text { 10 }\end{array}$ & $\begin{array}{c}\mathrm{AD} \\
\mathrm{I} \mathbf{I}\end{array}$ & $\mathrm{AE}$ \\
\hline $\mathrm{BA}$ & BB & $\begin{array}{c}\mathrm{BC} \\
2\end{array}$ & $\mathrm{BD}$ & $\mathrm{BE}$ \\
\hline $\mathrm{CA}$ & $\begin{array}{c}\mathrm{CB} \\
\mathrm{I}\end{array}$ & $\begin{array}{c}\mathrm{CC} \\
4\end{array}$ & $\begin{array}{c}\text { CD } \\
\text { I I }\end{array}$ & $\begin{array}{c}\mathrm{CE} \\
5\end{array}$ \\
\hline DA & $\mathrm{DB}$ & $\overline{\mathrm{DC}}$ & $\begin{array}{c}\mathrm{DD} \\
30\end{array}$ & $\begin{array}{l}\mathrm{DE} \\
\mathrm{II}\end{array}$ \\
\hline EA & EB & EC & $\mathrm{ED}$ & $\overline{\mathrm{EE}}$ \\
\hline
\end{tabular}

Tokushima (Japan) $\mathrm{n}=\mathrm{I} 09$

\begin{tabular}{|c|c|c|c|c|}
\hline $\begin{array}{l}\text { AA } \\
36\end{array}$ & $\begin{array}{c}\mathrm{AB} \\
\mathrm{I}\end{array}$ & $\begin{array}{c}A C \\
5\end{array}$ & $\begin{array}{c}\text { AD } \\
23\end{array}$ & $\begin{array}{c}\mathrm{AE} \\
\mathbf{2}\end{array}$ \\
\hline $\mathrm{BA}$ & $\overline{B B}$ & BC & BD & BE \\
\hline $\mathrm{CA}$ & $\overline{\mathrm{CB}}$ & $\mathrm{CC}$ & $\begin{array}{c}\mathrm{CD} \\
5\end{array}$ & $\begin{array}{c}\mathrm{CE} \\
\mathbf{2}\end{array}$ \\
\hline $\mathrm{DA}$ & DB & $\mathrm{DC}$ & $\begin{array}{c}\mathrm{DD} \\
8\end{array}$ & $\begin{array}{c}\mathrm{DE} \\
\mathrm{I} 6\end{array}$ \\
\hline EA & EB & $\mathrm{EC}$ & ED & $\overline{\mathrm{EE}}$ \\
\hline
\end{tabular}

Of the above results the following was also noted:

(a) Stoke Mandeville:

Compression Injuries

Flexion Rotation

No Bony Injury

(b) Royal Perth (Rehabilitation) Hospital:

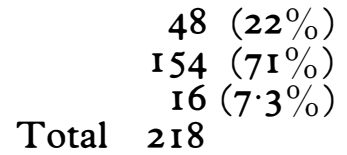

Compression

Flexion Rotation

Extension

(c) Tokushima:

Compression

Flexion Rotation

Hyperextension

(d) South West Region:

No details available
$48(33 \%)$

$72(50 \%)$

$24(17 \%)$

IO $(9 \%)$

$64(59 \%)$
$35(32 \%)$

As can be seen a fundamental difference was observed. The percentage 
of extension injuries was much greater in Tokushima-59\%, compared to $7.3 \%$ in Stoke Mandeville. In Tokushima, falls and other mobile causes, caused a greater percentage of the accidents. A comparison of the results before and after I973 (see Table VII) showed that regarding to neurological status of each group:

(I) The compression group showed almost the same incidence of initial complete tetraplegia in both series, $47.5 \%$ (before 1973 ) and $42 \%$ (after I973) whilst transition complete to incomplete (I6\% before I973) seven cases (15\% after 1973).

(2) The flexion rotation group is in the authors' opinion that group where surgical fusion will be used in a few cases of true late instability. This study showed one unstable injury in the compression group, five cases for flexion-rotation group I injuries, no cases flexion-rotation group II injuries and no cases in extension injuries. Total instability was thus present in six cases $(4 \cdot 2 \%)$ of 144 cases. The results before and after 1973 show 23 cases of a total of 380 cases an instability rate of $6 \%$. (see Table VI).

(3) As a corollary in studying the extension injuries, the clinical syndromes on admission were compared before 1973 to after I973. (see Table VIII) Numbers are too small to make major worthwhile observations.

Each study showed the predominance of the central cord lesion. Further studies as numbers are available are now possible as now reported in other areas of neurological result as noted in Tables IXa and X.

(4) Finally an attempt was made to study a correlation between reduction achieved and neurological change on recovery. (see Tables IX, IXb and X).

The guidelines of anatomical, residual deformity, partial or failed reduction are appended below in each table. From the material presented, the authors can only draw a few observations as the numbers are not yet adequate for better decision.

(I) It is noted that despite failure to reduce facetal dislocation (e.g. group I) neurological recovery proceeds. (see Table IXa).

TABLE VI

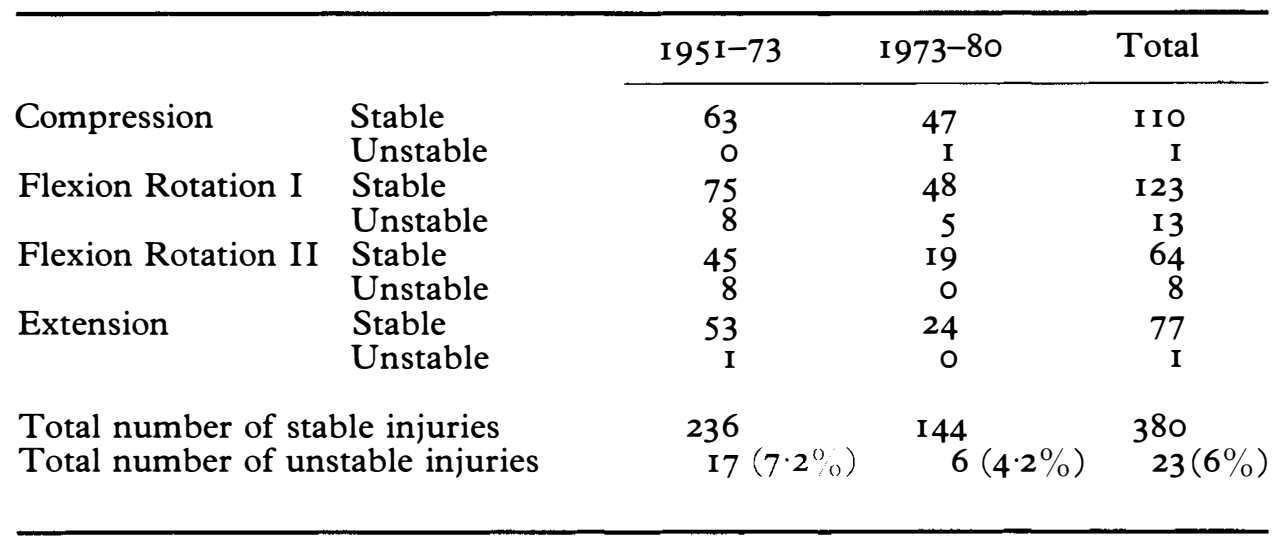




\section{TABLE VII}

Neurological State on Admission and Discharge I95 I-I973

\begin{tabular}{|c|c|c|c|c|}
\hline & & Admission & Discharge & $\begin{array}{l}\text { Complete/ } \\
\text { Incomplete }\end{array}$ \\
\hline Compression & $\begin{array}{l}\text { Complete } \\
\text { Incomplete }\end{array}$ & $\begin{array}{l}30=47 \cdot 5 \% \\
\frac{33}{63}=52 \cdot 5 \%\end{array}$ & $\begin{array}{l}20=32 \% \\
\frac{43}{63}=68 \%\end{array}$ & $\mathrm{IO}=\mathrm{I} 6 \%$ \\
\hline Flexion Rotation I & $\begin{array}{l}\text { Complete } \\
\text { Incomplete }\end{array}$ & $\begin{array}{l}17=23 \% \\
58=77 \% \\
\frac{75}{15}\end{array}$ & $\begin{array}{l}\mathrm{II}=\mathrm{I} 5 \% \\
64=85 \% \\
75\end{array}$ & $6=8 \%$ \\
\hline Flexion Rotation II & $\begin{array}{l}\text { Complete } \\
\text { Incomplete }\end{array}$ & $\begin{array}{l}25=55 \% \\
20=45 \% \\
\frac{45}{-}\end{array}$ & $\begin{array}{l}\mathrm{I} 4=3 \mathrm{I} \% \\
\frac{3 \mathrm{I}}{45}=69 \%\end{array}$ & $9=20 \%$ \\
\hline Extension & $\begin{array}{l}\text { Complete } \\
\text { Incomplete }\end{array}$ & $\begin{aligned} 9 & =17 \% \\
\frac{44}{53} & =83 \%\end{aligned}$ & $\begin{aligned} 6 & =\mathrm{II} \% \\
\frac{47}{53} & =89 \%\end{aligned}$ & $3=6 \%$ \\
\hline \multicolumn{5}{|c|}{ I973-I980 } \\
\hline Compression & $\begin{array}{l}\text { Complete } \\
\text { Incomplete }\end{array}$ & $\begin{array}{l}20=42 \% \\
\frac{28}{48}=58 \%\end{array}$ & $\begin{array}{l}13=27 \% \\
\frac{35}{48}=73 \%\end{array}$ & $7=15 \%$ \\
\hline Flexion Rotation I & $\begin{array}{l}\text { Complete } \\
\text { Incomplete }\end{array}$ & $\begin{aligned} 7 & =13 \% \\
46 & =87 \% \\
\frac{53}{23} & \end{aligned}$ & $\begin{aligned} 2 & =4 \% \\
\frac{51}{53} & =96 \%\end{aligned}$ & $5=9 \%$ \\
\hline Flexion Rotation II & $\begin{array}{l}\text { Complete } \\
\text { Incomplete }\end{array}$ & $\begin{aligned} \mathrm{IO} & =53 \% \\
\frac{9}{19} & =47 \%\end{aligned}$ & $\begin{aligned} & 9=47 \% \\
& \text { IO }=53 \% \\
& \frac{19}{19}\end{aligned}$ & $\mathrm{I}=5 \%$ \\
\hline Extension & $\begin{array}{l}\text { Complete } \\
\text { Incomplete }\end{array}$ & $\begin{aligned} 2 & =8 \% \\
22 & =92 \% \\
\frac{24}{-} & \end{aligned}$ & $\begin{aligned} \mathrm{I} & =4 \% \\
\frac{23}{24} & =96 \%\end{aligned}$ & $\mathrm{I}=4 \%$ \\
\hline
\end{tabular}

(2) Reduction of the bony injury has not been shown to be regularly necessary for neurological recovery in those cases with compression injuries (see table X) and flexion-rotation group I. (see Table IXa). Neurological recovery is not regularly dependant on attempts at reduction. In flexion rotation group II cases (see Table IXb) it would appear that more recovery 
TABLE VIII

\section{Extension Injuries of the Cervical Spine}

Perth, Western Australia

Clinical Syndromes on Admission

Central Cord Lesion

Anterior Cord Lesion

Spinal Cord Concussion

\begin{tabular}{|c|c|c|}
\hline I95I-73 & I973-80 & I $95 \mathrm{I}-80$ \\
\hline 23 & I3 & 36 \\
\hline 2 & 4 & 6 \\
\hline 8 & I & 9 \\
\hline 2 & 2 & 4 \\
\hline 9 & 3 & I I \\
\hline- & I & - \\
\hline 44 & 24 & 66 \\
\hline
\end{tabular}

\section{TABLE IXa}

Correlation Between the Degree of Reduction Achieved and Neurological Recovery

Flexion Rotation I

anatomical 23

\begin{tabular}{|c|c|c|c|c|}
\hline $\begin{array}{c}\text { AA } \\
\text { I }\end{array}$ & $\begin{array}{c}\mathrm{AB} \\
\mathrm{I}\end{array}$ & AC & $\mathrm{AD}$ & $\mathrm{AE}$ \\
\hline$\overline{\mathrm{BA}}$ & $\overline{\mathrm{BB}}$ & $\begin{array}{c}\text { BC } \\
\text { I }\end{array}$ & $\begin{array}{c}\text { BD } \\
\mathbf{I}\end{array}$ & $\mathrm{BE}$ \\
\hline CA & $\mathrm{CB}$ & $\overline{\mathrm{CC}}$ & $\mathrm{CD}$ & $\mathrm{CE}$ \\
\hline $\mathrm{DA}$ & $\begin{array}{c}\mathrm{DB} \\
\mathbf{I}\end{array}$ & $\overline{\mathrm{DC}}$ & $\begin{array}{c}\mathrm{DD} \\
\mathbf{I}\end{array}$ & $\begin{array}{c}\mathrm{DE} \\
\mathrm{I} 4\end{array}$ \\
\hline EA & EB & $\mathrm{EC}$ & $\overline{\mathrm{ED}}$ & $\overline{\mathrm{EE}}$ \\
\hline
\end{tabular}

Improvement $17 / 23=74 \%$

partial I4

\begin{tabular}{|c|c|c|c|c|}
\hline $\mathrm{AA}$ & $\begin{array}{c}\mathrm{AB} \\
\mathrm{I}\end{array}$ & $\mathrm{AC}$ & $\mathrm{AD}$ & $\mathrm{AE}$ \\
\hline $\mathrm{BA}$ & $\mathrm{BB}$ & $\mathrm{BC}$ & $\begin{array}{c}\mathrm{BD} \\
\mathbf{I}\end{array}$ & $\mathrm{BE}$ \\
\hline $\mathrm{CA}$ & $\mathrm{CB}$ & $\mathrm{CC}$ & $\begin{array}{c}\mathrm{CD} \\
\mathbf{I}\end{array}$ & $\begin{array}{c}\mathrm{CE} \\
\mathbf{I}\end{array}$ \\
\hline $\mathrm{DA}$ & $\mathrm{DB}$ & $\mathrm{DC}$ & $\begin{array}{c}\mathrm{DD} \\
\mathrm{I}\end{array}$ & $\begin{array}{c}\mathrm{DE} \\
8\end{array}$ \\
\hline $\mathrm{EA}$ & $\mathrm{EB}$ & $\mathrm{EC}$ & $\mathrm{ED}$ & $\begin{array}{c}\mathrm{EE} \\
\mathbf{I}\end{array}$ \\
\hline
\end{tabular}

Improvement I I $/$ I $4=78 \cdot 5 \%$ residual 7

\begin{tabular}{|c|c|c|c|c|}
\hline AA & $\begin{array}{c}\mathrm{AB} \\
\mathrm{I}\end{array}$ & $\begin{array}{c}\text { AC } \\
\text { I }\end{array}$ & $\mathrm{AD}$ & $\mathrm{AE}$ \\
\hline $\mathrm{BA}$ & $\overline{\mathrm{BB}}$ & $\mathrm{BC}$ & $\begin{array}{c}\text { BD } \\
\text { I }\end{array}$ & $\mathrm{BE}$ \\
\hline CA & $\mathrm{CB}$ & $\mathrm{CC}$ & \begin{tabular}{|c|}
$\mathrm{CD}$ \\
$\mathbf{I}$ \\
\end{tabular} & $\mathrm{CE}$ \\
\hline $\mathrm{DA}$ & DB & $\mathrm{DC}$ & $\mathrm{DD}$ & $\begin{array}{c}\mathrm{DE} \\
3\end{array}$ \\
\hline EA & EB & $\mathrm{EC}$ & ED & $\mathrm{EE}$ \\
\hline
\end{tabular}

failed 8

\begin{tabular}{|c|c|c|c|c|}
\hline $\mathrm{AA}$ & $\mathrm{AB}$ & $\mathrm{AC}$ & $\mathrm{AD}$ & $\mathrm{AE}$ \\
\hline $\mathrm{BA}$ & $\mathrm{BB}$ & $\mathrm{BC}$ & $\begin{array}{c}\mathrm{BD} \\
\mathrm{I}\end{array}$ & $\mathrm{BE}$ \\
\hline $\mathrm{CA}$ & $\mathrm{CB}$ & $\mathrm{CC}$ & $\begin{array}{c}\mathrm{CD} \\
\mathrm{I}\end{array}$ & $\mathrm{CE}$ \\
\hline $\mathrm{DA}$ & $\mathrm{DB}$ & $\mathrm{DC}$ & $\begin{array}{c}\mathrm{DD} \\
\mathrm{I}\end{array}$ & $\begin{array}{c}\mathrm{DE} \\
3\end{array}$ \\
\hline $\mathrm{EA}$ & $\mathrm{EB}$ & $\mathrm{EC}$ & $\mathrm{ED}$ & $\begin{array}{c}\mathrm{EE} \\
2\end{array}$ \\
\hline
\end{tabular}

Improvement $5 / 8=62 \%$

$\mathrm{n}=52$ 
Correlation Between the Degree of Reduction Achieved and Neurological Recovery

Flexion Rotation II

anatomical I 2

\begin{tabular}{|c|c|c|c|c|}
\hline $\begin{array}{c}\text { AA } \\
4\end{array}$ & $\begin{array}{c}\mathrm{AB} \\
\mathrm{I}\end{array}$ & $\mathrm{AC}$ & $\mathrm{AD}$ & $\overline{\mathrm{AE}}$ \\
\hline$\overline{\mathrm{BA}}$ & $\begin{array}{c}\text { BB } \\
\text { I }\end{array}$ & BC & $\begin{array}{c}\text { BD } \\
2\end{array}$ & $\mathrm{BE}$ \\
\hline CA & $\overline{\mathrm{CB}}$ & $\mathrm{CC}$ & CD & $\mathrm{CE}$ \\
\hline $\mathrm{DA}$ & DB & $\mathrm{DC}$ & DD & $\begin{array}{c}\mathrm{DE} \\
\mathbf{2}\end{array}$ \\
\hline EA & EB & EC & ED & $\mathrm{EE}$ \\
\hline
\end{tabular}

residual 2

Improvement $5 / 12=42^{\circ} \%$

\begin{tabular}{|c|c|c|c|c|}
\hline $\mathrm{AA}$ & $\mathrm{AB}$ & $\mathrm{AC}$ & $\mathrm{AD}$ & $\mathrm{AE}$ \\
\hline $\mathrm{BA}$ & $\mathrm{BB}$ & $\mathrm{BC}$ & $\mathrm{BD}$ & $\mathrm{BE}$ \\
\hline $\mathrm{CA}$ & $\mathrm{CB}$ & $\mathrm{CC}$ & $\mathrm{CD}$ & $\mathrm{CE}$ \\
\hline $\mathrm{DA}$ & $\mathrm{DB}$ & $\mathrm{DC}$ & $\mathrm{DD}$ & $\begin{array}{c}\mathrm{DE} \\
2\end{array}$ \\
\hline EA & $\mathrm{EB}$ & $\mathrm{EC}$ & $\mathrm{ED}$ & $\mathrm{EE}$ \\
\hline
\end{tabular}

Improvement $2 / 2=100 \%$

partial 3

\begin{tabular}{|c|c|c|c|c|}
\hline $\begin{array}{c}\mathrm{AA} \\
3\end{array}$ & $\mathrm{AB}$ & $\mathrm{AC}$ & $\mathrm{AD}$ & $\mathrm{AE}$ \\
\hline $\mathrm{BA}$ & $\mathrm{BB}$ & $\mathrm{BC}$ & $\mathrm{BD}$ & $\mathrm{BE}$ \\
\hline $\mathrm{CA}$ & $\mathrm{CB}$ & $\mathrm{CC}$ & $\mathrm{CD}$ & $\mathrm{CE}$ \\
\hline $\mathrm{DA}$ & $\mathrm{DB}$ & $\mathrm{DC}$ & $\mathrm{DD}$ & $\mathrm{DE}$ \\
\hline $\mathrm{EA}$ & $\mathrm{EB}$ & $\mathrm{EC}$ & $\mathrm{ED}$ & $\mathrm{EE}$ \\
\hline
\end{tabular}

failed I

\begin{tabular}{|c|c|c|c|c|}
\hline $\begin{array}{c}\mathrm{AA} \\
\mathrm{I}\end{array}$ & $\mathrm{AB}$ & $\mathrm{AC}$ & $\mathrm{AD}$ & $\mathrm{AE}$ \\
\cline { 1 - 2 } $\mathrm{BA}$ & $\mathrm{BB}$ & $\mathrm{BC}$ & $\mathrm{BD}$ & $\mathrm{BE}$ \\
\hline $\mathrm{CA}$ & $\mathrm{CB}$ & $\mathrm{CC}$ & $\mathrm{CD}$ & $\mathrm{CE}$ \\
\hline $\mathrm{DA}$ & $\mathrm{DB}$ & $\mathrm{DC}$ & $\mathrm{DD}$ & $\mathrm{DE}$ \\
\hline $\mathrm{EA}$ & $\mathrm{EB}$ & $\mathrm{EC}$ & $\mathrm{ED}$ & $\mathrm{EE}$ \\
\hline
\end{tabular}

$\mathrm{n}=\mathrm{I} 8$

occurred in those cases with anatomical reduction. Figures however are as yet not adequate.

(3) These observations would strengthen the widely held view that the fate of the neurological injury is largely determined at the time of the accident as held by many authors. (Kakulas, 1976; Bedbrook, I979; Frankel et al., 1977).

\section{Discussion}

The present study increases our clinical knowledge which can be applied to the management of cervical injuries with paralysis of spinal cord origin. The authors used the classification which has been well reported in the Proceedings of the Veterans Administration I9th Conference in 1973. There was a small group of cases where, only after detailed consideration, could a satisfactory classification be achieved, most were self evident. 
Correlation Between the Degree of Reduction Achieved and Neurological Recovery

Compression Injury anatomical 9

\begin{tabular}{|c|c|c|c|c|}
\hline $\begin{array}{c}\mathrm{AA} \\
3\end{array}$ & $\mathrm{AB}$ & $\mathrm{AC}$ & $\mathrm{AD}$ & $\mathrm{AE}$ \\
\hline $\mathrm{BA}$ & $\begin{array}{c}\mathrm{BB} \\
2\end{array}$ & $\mathrm{BC}$ & $\mathrm{BD}$ & $\mathrm{BE}$ \\
\hline $\mathrm{CA}$ & $\mathrm{CB}$ & $\mathrm{CC}$ & $\mathrm{CD}$ & $\mathrm{CE}$ \\
\hline $\mathrm{DA}$ & $\mathrm{DB}$ & $\mathrm{DC}$ & $\mathrm{DD}$ & $\mathrm{DE}$ \\
4
\end{tabular}

Improvement $4 / 9=44 \%$ partial I3

\begin{tabular}{|c|c|c|c|c|}
\hline $\begin{array}{c}\mathrm{AA} \\
7\end{array}$ & $\begin{array}{c}\mathrm{AB} \\
4\end{array}$ & $\mathrm{AC}$ & $\mathrm{AD}$ & $\mathrm{AE}$ \\
\hline $\mathrm{BA}$ & $\begin{array}{c}\mathrm{BB} \\
2\end{array}$ & $\mathrm{BC}$ & $\mathrm{BD}$ & $\mathrm{BE}$ \\
\hline $\mathrm{CA}$ & $\mathrm{CB}$ & $\mathrm{CC}$ & $\mathrm{CD}$ & $\mathrm{CE}$ \\
$\mathrm{IA}$ & $\mathrm{DB}$ & $\mathrm{DC}$ & $\mathrm{DD}$ & $\mathrm{DE}$ \\
\hline $\mathrm{EA}$ & $\mathrm{EB}$ & $\mathrm{EC}$ & $\mathrm{ED}$ & $\mathrm{EE}$ \\
\hline
\end{tabular}

Improvement $5 / 14=36^{\%} \%$ $\mathrm{n}=42$ residual 9

\begin{tabular}{|c|c|c|c|c|}
\hline $\mathrm{AA}$ & $\begin{array}{c}\mathrm{AB} \\
\mathrm{I}\end{array}$ & $\begin{array}{c}\mathrm{AC} \\
\mathrm{I}\end{array}$ & $\mathrm{AD}$ & $\mathrm{AE}$ \\
\hline $\mathrm{BA}$ & $\mathrm{BB}$ & $\mathrm{BC}$ & $\mathrm{BD}$ & $\mathrm{BE}$ \\
\hline $\mathrm{CA}$ & $\mathrm{CB}$ & $\mathrm{CC}$ & $\begin{array}{c}\mathrm{CD} \\
3\end{array}$ & $\begin{array}{c}\mathrm{CE} \\
\mathbf{I}\end{array}$ \\
\hline $\mathrm{DA}$ & $\mathrm{DB}$ & $\mathrm{DC}$ & $\begin{array}{c}\mathrm{DD} \\
\mathrm{I}\end{array}$ & $\begin{array}{c}\mathrm{DE} \\
2\end{array}$ \\
\hline $\mathrm{EA}$ & $\mathrm{EB}$ & $\mathrm{EC}$ & $\mathrm{ED}$ & $\mathrm{EE}$ \\
\hline
\end{tabular}

Improvement $8 / 9=89 \%$

failed I I

\begin{tabular}{|c|c|c|c|c|}
\hline $\begin{array}{c}\mathrm{AA} \\
4\end{array}$ & $\begin{array}{c}\mathrm{AB} \\
2\end{array}$ & $\mathrm{AC}$ & $\mathrm{AD}$ & $\mathrm{AE}$ \\
\hline $\mathrm{BA}$ & $\mathrm{BB}$ & $\begin{array}{c}\text { BC } \\
\text { I }\end{array}$ & $\mathrm{BD}$ & $\mathrm{BE}$ \\
\hline CA & $\mathrm{CB}$ & $\mathrm{CC}$ & $\begin{array}{c}\text { CD } \\
\text { I } \\
\end{array}$ & CE \\
\hline DA & DB & $\mathrm{DC}$ & $\mathrm{DD}$ & $\begin{array}{c}\mathrm{DE} \\
3\end{array}$ \\
\hline EA & EB & $\mathrm{EC}$ & $\mathrm{ED}$ & $\mathrm{EE}$ \\
\hline
\end{tabular}

Improvement $7 /$ I I $=64 \%$

Multiple forces are regularly responsible for the final bony manifestation, however we still feel that simplicity in reporting such, has much merit in our present state of knowledge. (White, I976, I978; Punjabi and colleagues, I976). These authors are opening discussions that will ultimately be interesting. The neurological findings of this survey confirms many of the surveys well documented by one of us already. (Bedbrook, I979).

This study extends information in two ways. First, material with different aetiological facts emerging from a review such as from Tokushima (Japan) (Takakki, Ikata, I979), where different progress is noted indicating the importance of estimating quantum of accidents clearly. Falls and falling appear to have a lower quantum of damage than gross motor vehicle trauma where in this geographical group of cases the neurological recovery varied from one centre to another. (see Tables IV and V).

Secondly, information emerged indicating I 8 out of 29 cases or $62 \%$ of the diving and surfing accidents showed compression rather than flexion 
TABLE XI

Review of Cervical Fractures and Fracture Dislocations Perth, Western Australia I 95 I-I 980

\begin{tabular}{|c|c|c|c|c|}
\hline \multicolumn{5}{|c|}{ Neurological State on Admission } \\
\hline \multirow[t]{2}{*}{ Compression } & $\begin{array}{l}\text { Complete } \\
\text { Incomplete }\end{array}$ & $\begin{array}{l}50=45 \% \\
6 \mathrm{I}=55 \%\end{array}$ & $\begin{array}{l}33=30 \% \\
78=70 \%\end{array}$ & \multirow[t]{2}{*}{$17=15 \%$} \\
\hline & & I I I & I I I & \\
\hline \multirow[t]{2}{*}{ Flexion Rotation I } & $\begin{array}{l}\text { Complete } \\
\text { Incomplete }\end{array}$ & $\begin{array}{r}24=\mathrm{I} 9 \% \\
\mathrm{IO} 4=81 \% \\
\end{array}$ & $\begin{array}{r}\text { I3 }=10 \% \\
\text { I I } 5=90 \%\end{array}$ & \multirow[t]{2}{*}{ II $=9 \%$} \\
\hline & & 128 & I 28 & \\
\hline \multirow[t]{2}{*}{ Flexion Rotation II } & $\begin{array}{l}\text { Complete } \\
\text { Incomplete }\end{array}$ & $\begin{array}{l}35=55 \% \\
29=45 \%\end{array}$ & $\begin{array}{l}23=36 \% \\
4 I=64 \%\end{array}$ & \multirow[t]{2}{*}{$12=19 \%$} \\
\hline & & 64 & 64 & \\
\hline \multirow[t]{2}{*}{ Extension } & $\begin{array}{l}\text { Complete } \\
\text { Incomplete }\end{array}$ & $\begin{array}{l}\text { II }=14 \% \\
66=86 \%\end{array}$ & $\begin{aligned} 7 & =9 \% \\
70 & =91 \%\end{aligned}$ & \multirow[t]{2}{*}{$4=5 \%$} \\
\hline & & 77 & 77 & \\
\hline
\end{tabular}

rotation injuries. Falls and falling on the other hand seem to cause a high percentage of hyperextension injuries, nine out of 24 or $37 \%$.

Thirdly, our attempt at reviewing the effect of reduction didn't confirm it of great effectiveness in either compression or group I accidents. This is but an observation confirming pathological facts well known from our pathological studies and well recorded by Vinken and Bruyn. (Kakulas and Bedbrook, 1976).

Finally we could make no comment on time in hospital and method of treatment. As the cases were reviewed we could only reach a firm conclusion that time in hospital reflected multiple and individual factors in each case and thus we could not agree with authors such as Dickson and his colleagues (1973, I978) as well as Bradford et al. (1977) who sought to so relate time under rehabilitation to a single factor in the method of care of the spinal injury.

This review confirms the fundamentally conservative yet vigorous approach used for over 25 years in the centre in Perth, Western Australia.

\section{SUMMARY}

This is a continuation of a study already reported by the senior author; it confirms the conclusions drawn therein of the fundamentally conservative postural methods of care. The new study gives strength to the importance of reduction of gross dislocations but shows that minor displacements seen in original X-rays do not impede neurological recovery.

\section{RÉSUMÉ}

Ceci est la suite du rapport par l'auteur principal; il re-affirme les conclusions fondamentales de la methode des soins de postions conservatrices. La nouvelle etude assure l'importance 
de la reduction des dislocations globales, mais montre que les dislocations mineures vues dans les reyons- $x$ n'empechent pas las guerison neurologique.

\section{ZUSAMMENFASSUNG}

Diese Arbeit ergänzt eine vom leitenden Autor bereits bekannt $=$ gegebene Studie. Sie bestätigt die daraus gezogenen Schlübe überdie im wesentlichen konervativen Körperhaltungs-Plfegemethoden. Auch betont sie wie Wichtig es ist, stärkere Verrenkungen zu reduzieren; Zeigt aber, da geringere Verrenkungen, die auf den originalen Röntgenbildern sichtbar sind, die neurologische Besserung nicht beeinträchtigen.

\section{REFERENCES}

BEDBRooK, G. M. (I973). Compression, Flexion and Extension Injuries of the Cervical Spine with Tetraplegia. Proceedings of the Igth Veterans Administration Spinal Cord Conference. Published February 1977.

Bedbrook, G. M. (I979). Spinal Injuries with Tetraplegia and Paraplegia. Fournal of Bone and foint Surgery, 6IB, No. 3. p. 267-284.

Bradford, D. S., Akabarnia, B. A., Winter, R. B. \& Seljeskog, E. L. (I977). Surgical Stabilization of Fracture and Fracture Dislocations of the Thoracic Spine. Spine, 2, p. I 85-196.

Burke, D. C. (I97I). Hyperextension Injuries of the Spine. Fournal of Bone and foint Surgery. 53B, No. I, p. 3.

Dickson, J. H., Harrington, P. R. \& ERwin, W. D. (1973). Harrington Instrumentation in the Fractured, Unstable Thoracic and Lumbar Spine. Texas Medicine, 6I(9). p. $9 \mathrm{I}-98$.

Dickson, J. H., Harrington, P. R. \& Erwin, W. D. (1978). Results of Reduction and Stabilization of the Severely Fractured Thoracic and Lumbar Spine. Fournal of Bone and foint Surgery, 60A. p. 799-805.

FRANKEL, H. L. et al. (1969). The Value of Postural Reduction in the Initial Management of Closed Injuries of the Spine with Paraplegia and Tetraplegia. Paraplegia, 7. Part I. p. I79-192.

FRANKEL, H. L. et al. (1977). Result of Conservative Treatment of Extension Injuries of the Cervical Spine with Tetraplegia. Proceedings of the 19th Veterans Administration Spinal Cord Injury Conference. Scottsdale, Arizona. (1973). p. 52-56.

Kakulas, B. A. \& Bedbrook, G. M. (1976). Pathology of Injuries of the Vertebral Column. In Handbook of Clinical Neurology, 25, p. 27-42.

TAKAKKI, IKaTA (1979). Early Treatment of Cervical Spine Injuries with TetraplegiaEvaluation of our Routine Methods and Anterior Fusion. Orthopaedic and Traumatic Surgery. 22, No. 8, p. 763-777.

White, A. A., Southwick, W. O. \& Punjabi, M. M. (1976). Clinical Instability in the Lower Cervical Spine. Spine, I, p. I 5-27.

White, A. A. \& Punjabi, M. (1978). Clinical Biomechanics of the Spine. Leppencott. 